\title{
Joint Discussion 8 Solar and stellar activity cycles
}

\author{
Alexander G. Kosovichev ${ }^{1}$ and Klaus G. Strassmeier ${ }^{2}$ (eds.) \\ ${ }^{1}$ W.W. Hansen Experimental Physics Laboratory, Stanford University, Stanford, CA \\ 94305-4085, USA \\ email: sasha@quake.stanford.edu \\ ${ }^{2}$ Astrophysical Institute Potsdam, D-14482 Potsdam, Germany \\ email: kstrassmeier@aip.de
}

\section{Preface}

The solar magnetic field and its associated atmospheric activity exhibits periodic variations on a number of time scales. The 11-year sunspot cycle and its underlying 22-year magnetic cycle are, besides the 5-minute oscillation, the most widely known. Amplitudes and periods range from a few parts per million (ppm) and 2-3 minutes for p-modes in sunspots, a few $10 \mathrm{ppm}$ and 10 minutes for the granulation turn around, a few $100 \mathrm{ppm}$ and weeks for the lifetime of plages and faculae, $1000 \mathrm{ppm}$ and 27 days for the rotational signal from spots, to the long-term cycles of $90 \mathrm{yr}$ (Gleissberg cycle), 200-300 yr (Wolf, Spörer, Maunder minima), 2,400 yr from ${ }^{14} C$ tree-ring data, and possibly in excess of $100,000 \mathrm{yr}$.

The enormous complexity of atmospheric structure as observed on the Sun and some other cool stars makes it hard, if not impossible, to compare the Sun with 'solar-type

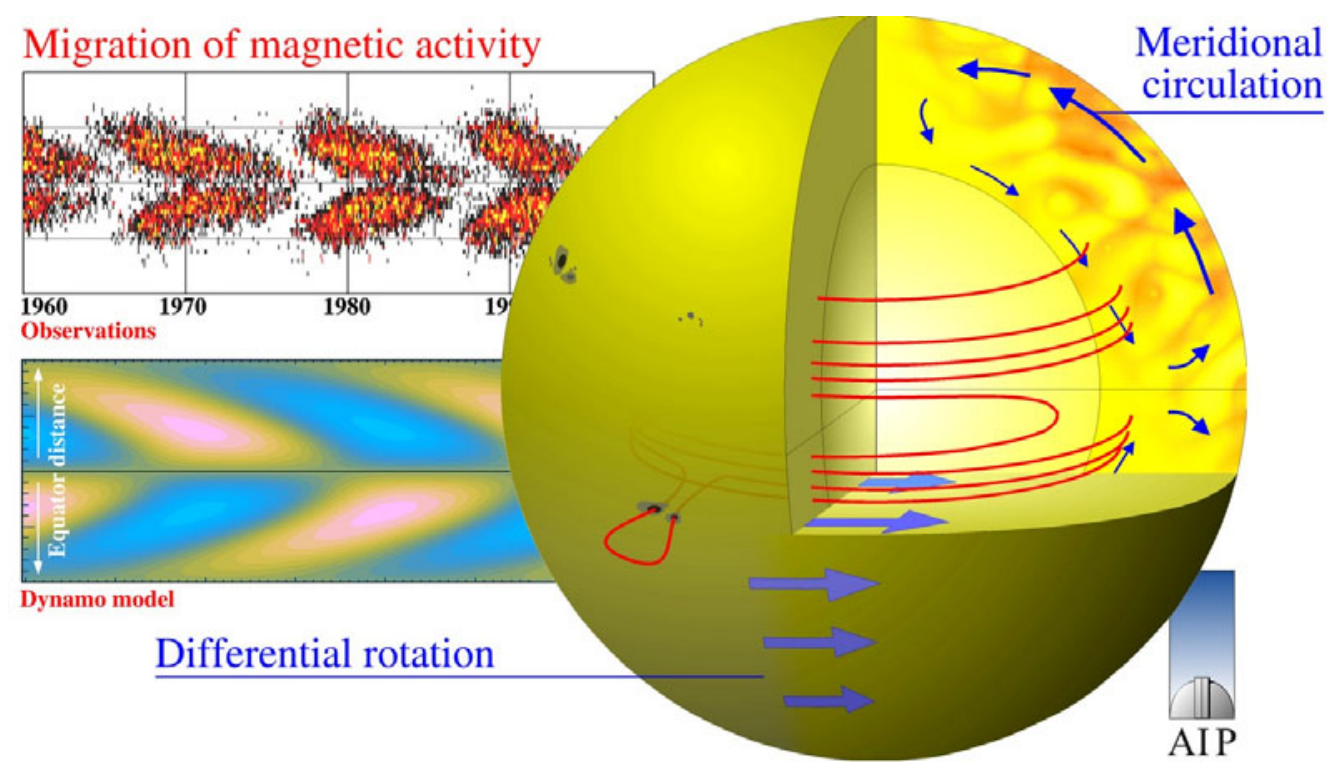

Figure 1. A graphical summary of some of the processes of magnetic activity and its observables. Courtesy of R. Arlt, AIP. 
stars' in great detail. Clearly, we need to identify parameters that can be observed and interpreted unambiguously. The most accepted dynamo signature is the presence of an activity cycle, well documented for the Sun as well as for solar-type stars by the Mount Wilson H\&K project. Just about recently, we are also detecting differential rotation and meridional flows on other stars. Again, the picture is not unambiguous yet, despite that there is just a single main ingredient that acts as the driving mechanism for activity in all atmospheric layers and the convective envelope: the dynamo-driven magnetic field.

Therefore, a Joint Discussion (JD) on the topic of "Solar and Stellar Activity Cycles" was held during the IAU General Assembly in Prague on August 17-18, 2006. Organized by Divisions II and IV with participating commissions 10, 12, 29, 35, 36, and 49, the meeting was made up by 18 invited talks in three subtopics. A total of approximately 120 astronomers participated in this one-and-a-half day gathering with many and lively discussions.

\section{Scientific Organizing Committee}

Alexander Kosovichev (USA) (co-chair), Klaus G. Strassmeier (Germany) (co-chair), Pavel Ambroz (Czech Republic), Martin Asplund (Australia), Svetlana V. Berduygina (Switzerland), Andrew Collier Cameron (UK), Dainis Dravins (Sweden), Lidia van DrielGesztelyi (France), Cheng Fang (China), John D. Landstreet (Canada), Valentin I. Makarov (Russia), Gautier Mathys (Chile), Roberto Pallavicini (Italy), Fernando MorenoInsertis (Spain), Takashi Sakurai (Japan), and Parameswaran Venkatakrishnan (India). We thank all of them for their help and many suggestions.

We thank all of the SOC members for their help and many suggestions. Special thanks go to our chairmen Nicolai Piskunov and John Landstreet who kept a strict eye on the watch. Rainer Arlt from the AIP produced and maintained the meeting web site and Katrin Götz managed the LOC and related issues.

Sasha Kosovichev and Klaus Strassmeier, co-chairs SOC, Potsdam, October 30, 2006 